\title{
Intuitive Human-Device Interaction for Video Control and Feedback
}

\author{
Toon De Pessemier, Luc Martens and Wout Joseph \\ imec - WAVES - Ghent University \\ Technologiepark-Zwijnaarde 15 \\ 9052 Ghent, Belgium \\ Email: toon.depessemier@ugent.be luc1.martens@ugent.be wout.joseph@ugent.be
}

\begin{abstract}
Current video services are still controlled in an old-fashioned way using keyboard and mouse for computers and remote control for $\mathrm{TV}$ sets. This paper presents more attractive and intuitive interaction methods using commercially available motion sensing input devices. These devices are typically based on a webcam-style add-on peripheral, thereby enabling facial recognition, gesture control, and speech recognition. These technologies are applied in this research to automatically authenticate a user, enable video control (play, pause, seeking), and browsing, selecting, and rating content by hand gestures or voice commands. By monitoring the user's gaze and using emotion recognition techniques, the user's interests and engagement with the content can be estimated. This is interpreted as implicit user feedback for the video content, and establishes an automatic feedback channel, which can be used for content personalization and recommendation. User tests showed an accurate recognition of voice and gestures and confirmed the attractiveness and intuitiveness of these techniques for end-users.
\end{abstract}

\section{INTRODUCTION}

Various initiatives have improved the human-computer interaction over the last decade. New technologies, such as virtual reality and motion sensing, enable qualitatively different interfaces and implicitly deriving user feedback. Detecting the body position and gaze (i.e. the direction of face/eyes) of people can provide insights into the level of attention or interest. Studies have shown a strong correlation between aspects of body language, such as body lean and the orientation of the head, and the attention payed to what is being said [1]. Disengagement and frustration may coincide with closer postural positions and more movement, whereas focused attention and less frustration occur with more distant, stable postural positions [2]. Systematic observation of people's movements in a specific context has various applications, e.g., assessing disruptive and off-task classroom behavior [3].

Various methods have been proposed to detect body position. For example, pressure sensors have been used for detecting a lean back position. Unfortunately, such an intrusive measurement method has shown to influence people's behavior [3]. Physical contact with sensors makes users aware of being tracked, thereby changing their behavior. Moreover, pressure sensors are not accurate enough: various degrees of leaning back or the curvature of the person's back cannot be tracked. Therefore, cameras are a better solution to track body position and head poses to estimate people's attention [4].
In addition, cameras allow to derive much more information, such as emotions visible on people's face. Action Units (AU) are the most important characteristic to classify emotions. AU are contractions or relaxations of one or multiple facial muscles. Research showed that emotions are cross-cultural and can be identified by a test panel by judging facial expressions due to AU [5]. These AU are also used by algorithms for the automatic detection of emotions. For example, sadness is typically characterized by a lower position of the mounth angles and eyebrows, as well as eyebrows closer to the eyes. Many commercial applications for emotion recognition exist, such as Face++ [6] and SkyBiometry [7]. However, these services, often available as a RESTful API service, typically expect a static picture with a frontal view of a person as input, whereas in realistic applications, cameras capture moving images of people [8]. Alternative API solutions that can handle video as input, such as Kairos emotion analysis API [9], are often expensive after a trial period. In this study, emotion recognition is based on an open source library that processes short videos of people on client side.

Six basic emotions are often distinguished: surprise, sad, happy, fear, disgust, and angry [5]. Subsequent research coupled the emotions to positive and negative counterparts: e.g., happy vs. sad or angry vs. fear [10]. Alternative models map emotions to continuous values. Based on this, most emotions can be interpreted as the combination of two orthogonal dimensions: valence ranging from unpleasant to pleasant, and arousal ranging from deactivation to activation [11]. In conclusion, many studies investigated human emotions, often with the goal of identifying typical features for emotions.

In this research, the Microsoft Kinect hardware (version 2) and the associated SDK [12] developed by Microsoft were used as sensing input and combined with a PC for processing the input and a TV screen as visual interface to the user. In comparison to version 1 (2010), the Kinect version 2 (2013) has a higher resolution camera allowing a more accurate recognition, which is required e.g., for gesture control. The Kinect allows to detect, amongst others, head orientation, body position, head engagement and looking away, lean forward, lean backward, gaze, sound, and movements. Through an adapter, the Kinect device is connected to a Windows machine, running a Video On Demand (VOD) application with Graphical User Interface (GUI) that is developed in Microsoft Visual 
Studio. Using this setup, the goal of this research is to evaluate the user experience obtained with various intuitive humandevice interaction methods. With these interaction methods, this paper presents an innovative control mechanism and feedback channel, which can be used for personalization. More specifically, the Kinect is used to control the user interface of a demo video delivering system (Section II), to derive implicit feedback for the content based on the user's body position and expressed emotions (Section III), and to provide explicit user feedback for the content (Section IV).

\section{INTUITIVE USER INTERFACE}

\section{A. Speech Recognition}

Speech recognition is based on the Speech Basics-WPF project [13], which allows to create a list of name-value couples for the speech commands. The name stands for a word or a sentence that has to be recognized, and the value is used for executing the associated processing code. By using the built-in speech recognition of the Kinect, training of the voice commands by every user is not necessary. To avoid false positives in the recognition process, e.g. due to the audio channel of the video, the keyword "Kinect" is used as prefix for all names of voice commands. Various commands are made available for browsing through the content: "Kinect scroll home", with variations, "left", "right", "down", or "up". The command "Kinect" + "play", "stop", "rewind" or "forward" is used for video playback control.

\section{B. Text-to-speech}

In combination with speech recognition, text-to-speech techniques make the human-computer interaction more intuitive. Text-to-speech does not require a camera or sensor device and is a natural feedback channel, whereas textual feedback would be difficult to read on a TV screen, especially when the user has to move his head in different directions for building a face model (Section II-E). The SpeechSynthesizer class [14] allows to convert text into speech with the desired volume, voice, and speed. Text-to-speech is used to provide feedback to the user while controlling the video playback and during the face recognition process.

\section{Hand Pointer}

Similar to the usage of a mouse, a virtual hand pointer is created by tracking the position of the user's hand and mapping this to the position of a cursor on the screen. Traditional mouse actions are translated into movements of the hand. A mouse click is translated into a hand tap, enabling to control buttons and links. These hand taps can also be used to select a number of stars (which are clickable objects shown on the screen) as feedback for the content. A mouse scroll is performed by a grab and straight movement of the hand. This can be used to scroll through the content library or during video playback to rewind or fast forward the video. If desired, two persons can control the user interface simultaneously, each with their own hand pointer. In contrast, a classic remote control allows only one person to control the device, although watching TV typically is a social activity involving multiple persons [15].

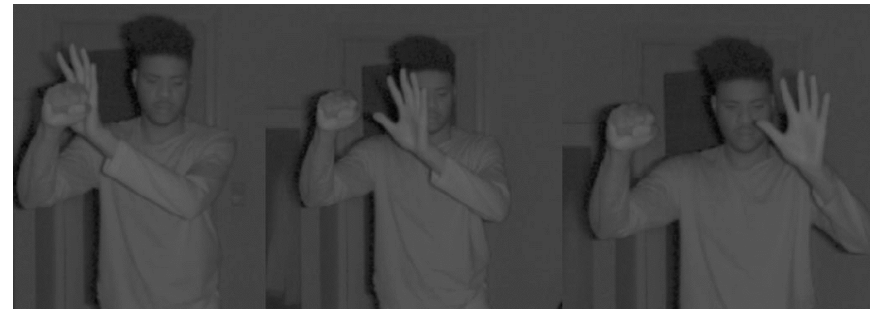

Figure 1. Scrolling through the content using gesture control. The right hand is used as a reference, while the left hand is used to wave thereby browsing through the content.

\section{Gesture Control}

Gesture Control is based on the Gestures-WPF project [16], which allows recognition of basis hand gestures. The gesture database is built upon labeled training videos of the gestures that have to be recognized. For each of these training videos recorded using the Kinect, the start and end frame of the hand gesture are marked. Recognition of these gestures is based on distinctive features, such as the speed of movement or the direction. In our intuitive interface, gesture control is used to browse through the content, as illustrated in Figure 1. One hand is used as a reference, while the other is used to browse through the user interface.

\section{E. User Identification}

Instead of the traditional user/password login procedure, user authentication can be automated using facial recognition techniques. Many solutions such as Face++ [6] or SkyBiometry [7] identify users based on two-dimensional pictures, thereby not fully utilizing the 3D potential of the Kinect. In contrast, the Sacknet FacialRecognition project [17] that we used, is based on the Kinect 3D Face Model Builder. This Face Model Builder measures various features of the face using the 3D capabilities of the camera for accuracy. Optimal face models are obtained in a room with a lot of light and distance of about 2.5 meters to the Kinect. To build such as model, users receive instructions for posing their head in various positions (front facing, side view). After building the face model, the identified features are compared to the features recognized during previous login procedures. In case of a match, the authentication process considers the user as recognized.

\section{F. Context Detection}

During usage of the video delivering system, sounds in the room are detected using the AudioBasic-WPF project [12]. This allows to derive the location of the sound source in the room based on the angle of arrival. A noise environment may distract the user. For speech recognition, only the sound source with the highest amplitude is used, and all other sounds are filtered out. 


\section{IMPLICIT USER FEEDBACK}

\section{A. Body Position}

Using the Kinect sensor, the three-dimensional position of the head and shoulders, body- and side lean, and the graze can be detected. The body and head position provide contextual information that can be used to estimate user interests, activities, and attention level. The Kinect SDK also provides properties such as "looking away", which gives an estimation of the orientation of the head w.r.t the Kinect, and "engagement", which is a combination of the looking away property and the eyes state (opened or closed).

\section{B. Emotion Recognition}

The six basic emotions [18] (surprise, sad, happy, fear, disgust, and angry) can be recognized based on AU. Although not all AU can be detected by the Kinect, studies have shown that $17 \mathrm{AU}$, which are available through the Kinect, are sufficient for accurate emotion recognition [19]. Similar to that study, we used the same $17 \mathrm{AU}$ that are available through the face tracking API of the Kinect SDK. The recognition of these AU by the Kinect results in a set of weights, which are stored and processed using the Pandas library [20]. Pandas is an open source Python library providing high-performance, easy-to-use data structures and data analysis tools. The weights of the 17 $\mathrm{AU}$ are stored into vectors for handling by machine learning techniques.

To get more insights into the raw data, Principal Component Analysis (PCA) is applied to these vectors of weights. Figure 2 shows the results of PCA, which reduces the 17 features to 2 features for visualization purpose. This clearly shows different clusters for different emotions, which indicates the potential for classification of the emotions based on AU.

The vectors containing 17 features are used to classify the video samples into one of the six emotion classes. For the classification, we use the Scikit learn library [21], which offers various machine learning functionality in Python. Two classification techniques are used: K-Nearest Neighbors (KNN) and Support Vector Machines (SVM). For KNN, the parameter K has to be specified. $\mathrm{K}$ is the number of nearest neighbors that are searched for each sample in order to classify it. For SVM, two parameters have to be specified. The $\gamma$ parameter defines how far the influence of a single training example reaches, with low values meaning 'far' and high values meaning 'close' [22]. The C parameter trades off misclassification of training examples against simplicity of the decision surface. A low $\mathrm{C}$ makes the decision surface smooth, while a high $\mathrm{C}$ aims at classifying all training examples correctly by giving the model freedom to select more samples as support vectors. The parameter values are determined by empirical evaluation and listed in Table I.

\section{EXPlicit User FeEdback}

As alternative for the traditional way of a mouse click on a star, various interactive ways for providing feedback for the content are proposed. Integrated with the way in which the user interface is controlled, users can provide feedback for

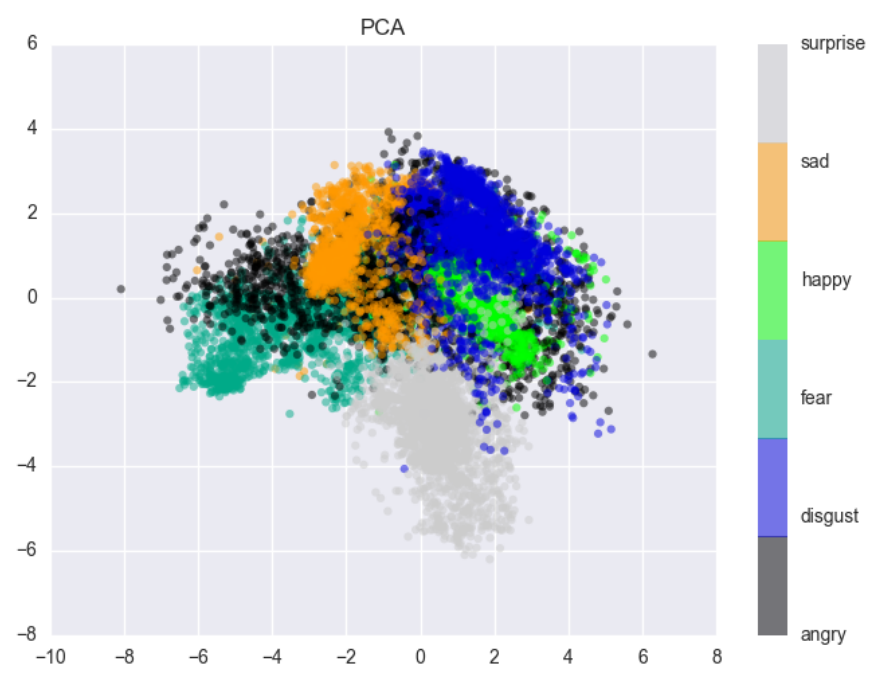

Figure 2. The six types of emotions in a two-dimensional space after Principal Component Analysis.

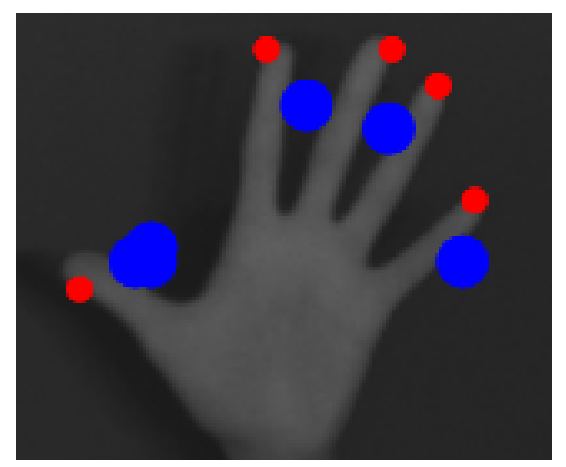

Figure 3. The finger tracking process used to allow users to give feedback.

the content by using the hand pointer approach (Section II-C). Besides, feedback can be provided in the following ways:

\section{A. Finger Tracking}

The Kinect SDK provides information about the user's body position, including the hands, but without the position of the fingers. Therefore, a finger recognition module was designed to detect the fingers of the user in front of the camera. The goal is to recognize how many fingers the user raises in order to provide feedback for the content. This module is based on the Lightbus Vitruvius FingerTracking project [23], which tracks fingers of both hands. The recognition process is focusing on the area near the hand (wrist), and computes the convex hull, which tremendously reduces the search space. Based on the convex hull, the positions of the fingers are estimated, as illustrated in Figure 3. Finger tracking is used as explicit feedback mechanism. This way, users can provide feedback by raising the hand(s) above the head while showing 1,2 , 3,4 or 5 fingers per hand, which triggers the recognition process. After a successful feedback registration, the user gets a confirmations on the screen and can lower the hand(s). 
Table I

ACCURACY OF THE CLASSIFICATION INTO SIX EMOTIONS

\begin{tabular}{l|l|l} 
Classification technique & Parameters & Accuracy \\
\hline K-Nearest Neighbors & $K=4$ & 0.9076 \\
Support Vector Machines & $\gamma=0.56, C=10$ & 0.9285 \\
\hline
\end{tabular}

\section{B. Drag and drop}

For the "Drag and Drop" method, the hand pointer is used to move a virtual rectangular object into the position of the desired score. This rectangle acts as the current score selection and can be moved to one of the areas on the screen that represents a specific feedback score.

\section{Hand Writing}

Users can provide a score by "writing" the number in the air with their finger. This hand writing gesture is recognized similarly to the gesture control method (Section II-D).

\section{Speech Recognition}

Besides browsing through content and video playback control, speech recognition can be used also as a feedback channel. Users can provide a rating by pronouncing the command "Kinect [number] star" where number is a score between one and five. So, this command can be used to express personal interests or appreciation for a video.

\section{Evaluation by User Tests}

The various interaction methods are evaluated in terms of accuracy and user experience by a test panel. The test panel consisted of 14 college students. Before the actual test, they received a short manual with instructions on how to use the intuitive interaction system, and a live demonstration illustrated this more in detail. These students were divided in groups of two persons, and could use the system during half an our. Figure 4 shows a screenshot of the user interface of this system. Afterwards, the test panel was asked to share their feelings and thoughts through a questionnaire. For the intuitive interaction methods, we assessed for qualitative aspects, such as easy of use and user experience. The explicit feedback mechanisms are evaluated in terms of accuracy by comparing the rating that a user intents to give, and the rating that was registered by the system. So, users were asked to provide a number of ratings ranging from 1 to 5 using the various methods. The results of these evaluations are visualized by a confusion matrix, which indicates which ratings are correctly (on the diagonal) or incorrectly (not on the diagonal) interpreted.

\section{A. Intuitive Interaction}

During the evaluation, users could try the various interaction mechanisms for browsing and selecting content. They indicated in the questionnaire to prefer the hand pointer (first choice) and speech recognition (second choice) technique. As a reason for this, users mentioned the ease of use and intuitive manner of interaction. Text-to-speech was appreciated as feedback channel. Moreover, test users requested to receive even more feedback, e.g., regarding the state of the recognition process (success, failure, calculating).

\section{B. User Identification}

Facial recognition using the Kinect 3D technique takes a lot of time, and requests users to pose in front of the camera in different positions (frontal view, side view, etc.). However, this pays off, since we experienced not a single false detection during the evaluation. Although users experience the Face Model building as tedious, they appreciate the corresponding security of this authentication process.

\section{Emotion Recognition}

The classification techniques for emotion recognition are evaluated on a data set with about 1260 video samples of people expressing emotions for each of the six classes. Because of practical reasons and the requirement of training the model, videos for emotion recognition were not recorded during the user test. To train and test the classifiers, 10-fold cross validation is used. The output of the classification is one of the six emotions or a probability for each of the six emotions. Table I shows the resulting accuracy, which is defined as the ratio of correct classifications and the total number of classifications.

\section{Finger Tracking}

The evaluation of the Finger Tracking method showed that the accuracy could be improved by using the distance from the hand to the Kinect in the finger recognition process. The optimal distance between the user and the Kinect sensor is ranging from 1 to 2.5 meter. Figure 5a shows the confusion matrix resulting from the test with users within this optimal distance range. The diagonal of the confusion matrix illustrates that accurate results are obtained: 90 of the 99 cases are correctly classified. For users who are further away from the Kinect, the accuracy of the Finger Tracking method decreases, as shown in Figure 5b. For this distance, 47 of the 90 cases are correctly classified. However, for most misclassifications, the error is limited to one finger. For the evaluation of the Finger Tracking, the detection of zero fingers is included as a separate class in the confusion matrices.

\section{E. Drag and Drop}

For providing explicit feedback, users indicated in the questionnaire to prefer the Drag and Drop method because of the accuracy, and quick and smooth usage. When using the Drag and Drop method to provide explicit ratings, users receive visual feedback on the screen during the "drag" phase. As a result, users correct their movements, if necessary, to select the desired rating value. The subjective feedback of the users emphasized the easy of use and accuracy of this method.

\section{F. Hand Writing}

Figure 6 shows the confusion matrix of the evaluation of the Hand Writing method. Using this method, 30 of the 48 cases are correctly classified. The Hand Writing method resulted in a rather low recognition accuracy, even within a short 


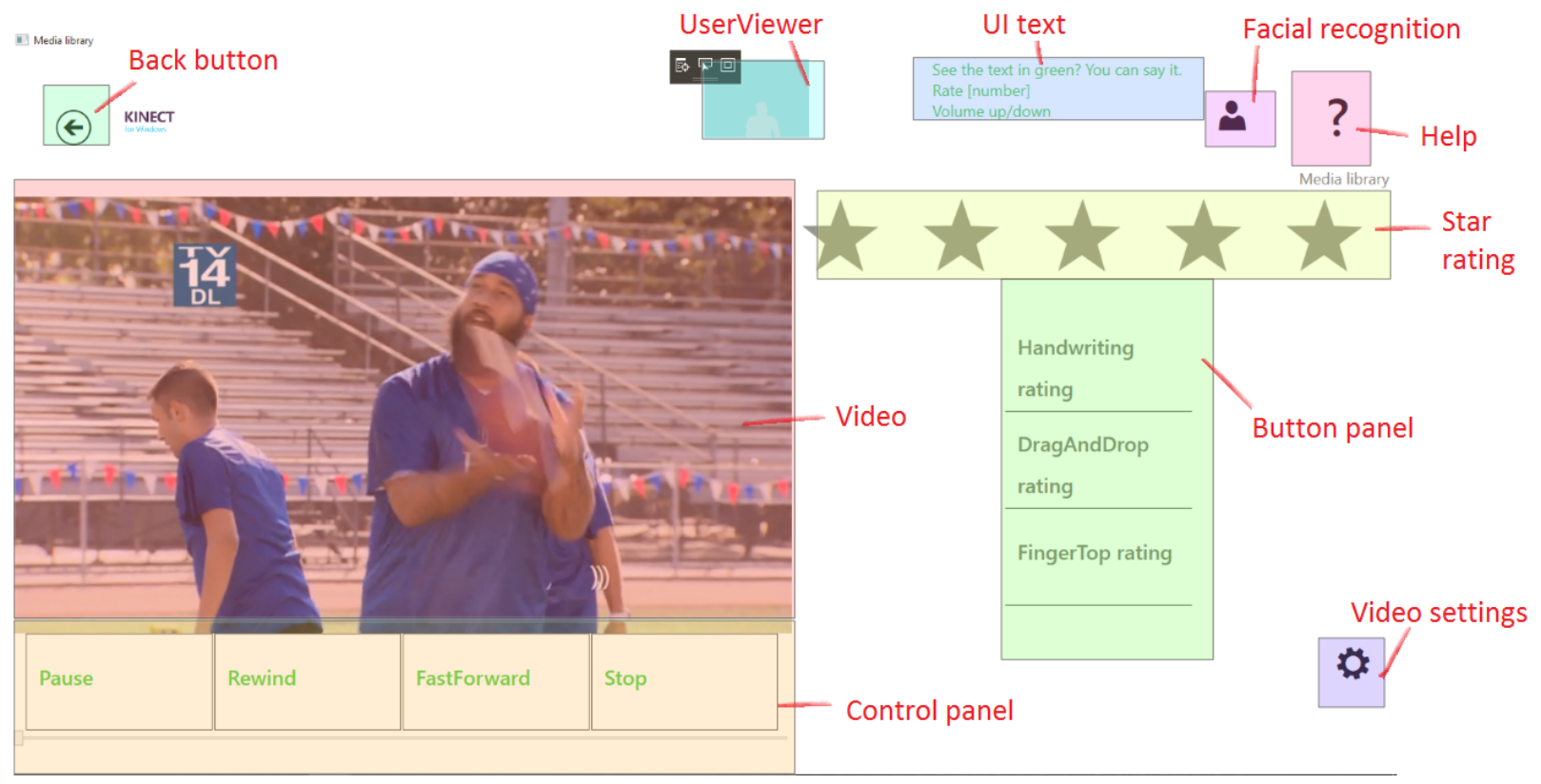

Figure 4. A Screenshot of the intuitive user interface.

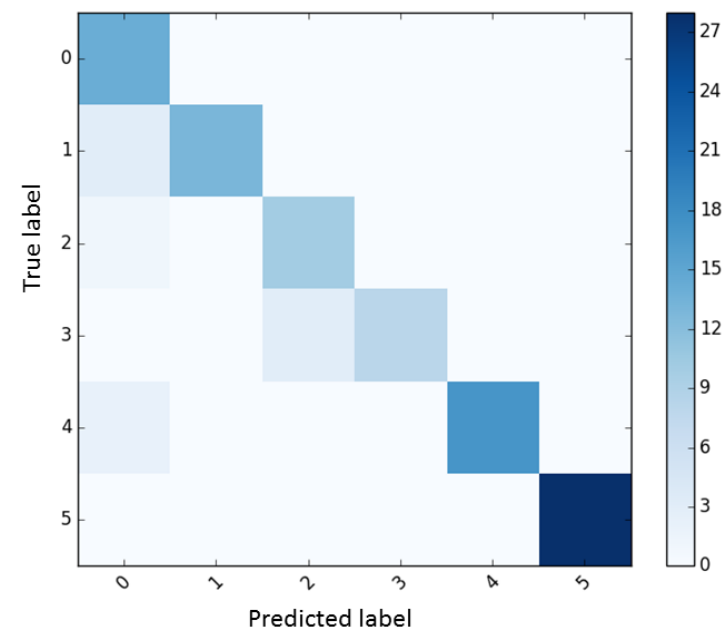

(a) The user is within a distance of 1 to 2.5 meters from the Kinect

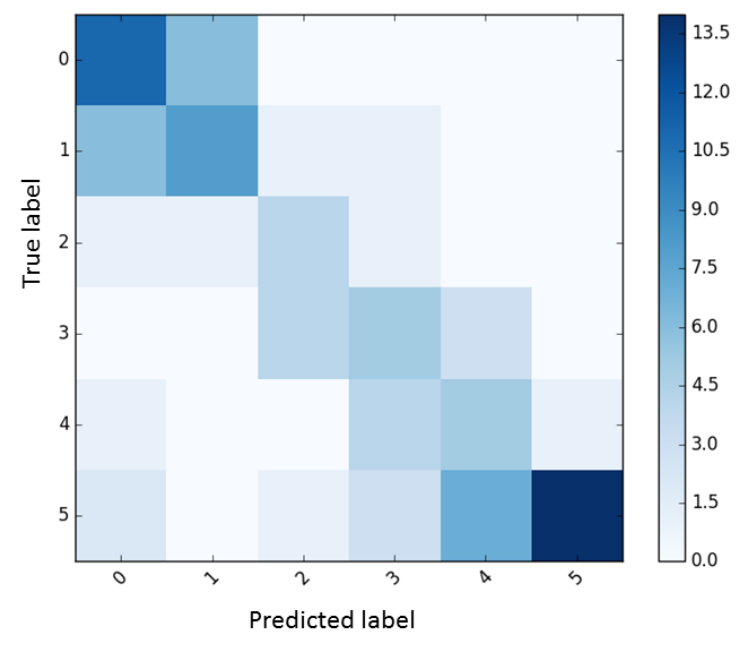

(b) The user is further than 2.5 meters away from the Kinect

Figure 5. Confusion matrices of the evaluation of the Finger Tracking method.

distance $(<2.5$ meter $)$ from the Kinect, since these gestures showed to be too similar. Moreover, the recognition process has difficulties to determine the begin and end of a hand writing gesture.

\section{G. Speech Recognition}

Figure 7a shows the confusion matrix resulting from providing explicit ratings using the Speech Recognition technique within a range of 3.5 meter from the Kinect. Voice commands that are not ratings, are classified in the class 'nothing'. Using speech recognition for providing explicit ratings proves to give accurate results: 114 of the 126 cases are correctly classified. Although the results are obtained with a muted video, voice commands withing this distance can even be recognized during video playback with audio. If the user is further than 3.5 meters away, the accuracy of speech recognition drops, as shown in Figure $7 \mathrm{~b}$, and muting the video becomes more important. For this distance, 80 of the 136 cases are correctly classified.

\section{CONCLUSION}

This paper proves the usability of intuitive interaction methods for control and browsing in video delivery systems. Speech recognition and various gestures are proposed and evaluated as interaction method and explicit feedback channel (i.e. for specifying a preference score for the content). Recognition of the user's emotions and body position is used to derive implicit 


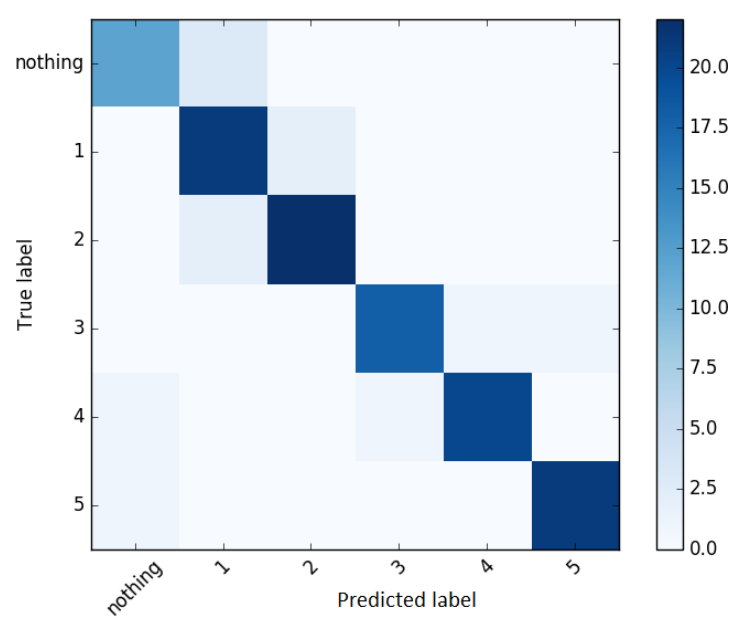

(a) The user is within a distance of 3.5 meters from the Kinect

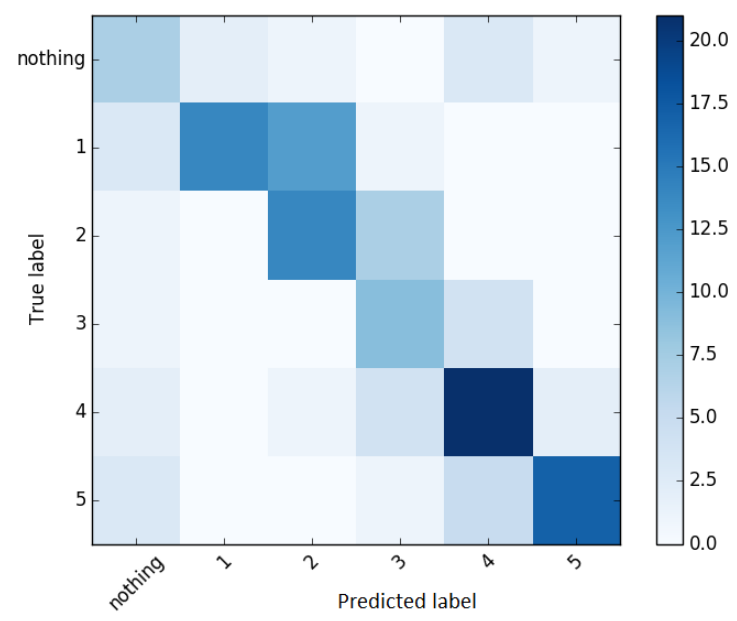

(b) The user is further than 3.5 meters away from the Kinect

Figure 7. Confusion matrices of the evaluation of the Speech Recognition method.

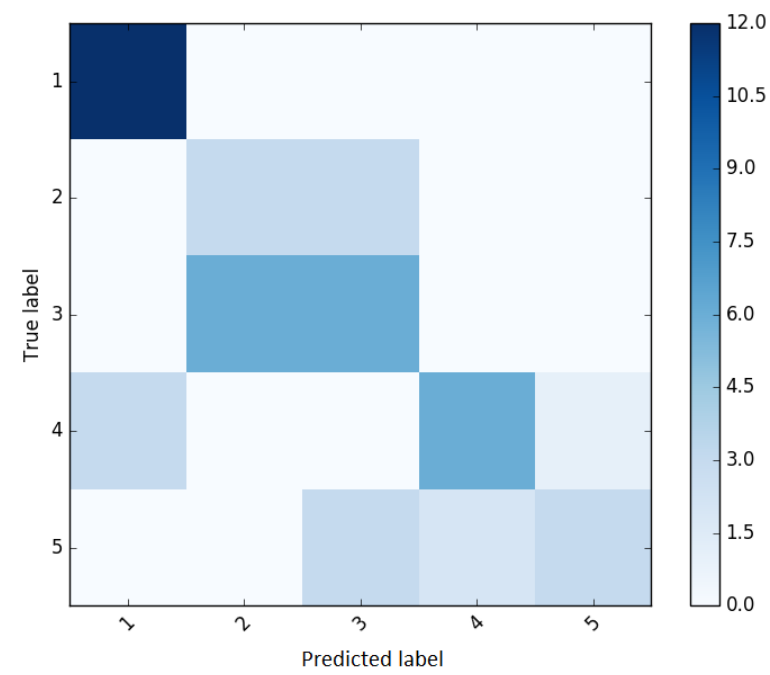

Figure 6. Confusion matrix of the evaluation of the Hand Writing method.

feedback. Evaluation with a test panel showed that users prefer to use the hand as a pointer and speech recognition as interaction methods. For providing explicit feedback, methods based on gestures (drag and drop) and speech recognition proved to be accurate and easy to use. A large distance between the user and the Kinect, as well as background noise have a negative influence on the accuracy.

These intuitive feedback mechanisms can be used in recommender systems to derive the user's personal preferences. Since the amount of user feedback is often limited in a classic setup (with remote control, or keyboard / mouse), these innovative feedback gathering and interaction methods can enhance the human-computer interaction channel, thereby improving the user experience and boosting the accuracy of recommender systems.

\section{ACKNOWLEDGMENT}

The authors would like to thank Miguel De Strooper for his extensive research and implementation work in the context of this study.

\section{REFERENCES}

[1] H. Lewis, Body language: A guide for professionals. SAGE Publications India, 2012.

[2] J. F. Grafsgaard, K. E. Boyer, E. N. Wiebe, and J. C. Lester, "Analyzing posture and affect in task-oriented tutoring." in FLAIRS Conference, 2012.

[3] W. F. Horn, C. K. Conners, K. C. Wells, and D. Shaw, "Use of the abikoff classroom observation coding system on a children's inpatient psychiatric unit," Journal of psychopathology and behavioral assessment, vol. 8, no. 1, pp. 9-23, 1986.

[4] R. Stiefelhagen, "Tracking focus of attention in meetings," in Proceedings of the 4th IEEE International Conference on Multimodal Interfaces. IEEE Computer Society, 2002, p. 273.

[5] P. Ekman, W. V. Friesen, M. O'Sullivan, A. Chan, I. DiacoyanniTarlatzis, K. Heider, R. Krause, W. A. LeCompte, T. Pitcairn, P. E. Ricci-Bitti, K. Scherer, M. Tomita, and A. Tzavaras, "Universals and cultural differences in the judgments of facial expressions of emotion," Journal of Personality and Social Psychology, vol. 53, no. 4, pp. 712717, 1987.

[6] Face++, "Leading face recognition on cloud," 2017, online Available at https://http://www.faceplusplus.com/.

[7] SkyBiometry, "Cloud-based face detection and recognition api," 2017, online Available at https://www.skybiometry.com/.

[8] T. De Pessemier, D. Verlee, and L. Martens, "Enhancing recommender systems for tv by face recognition," in 12th International Conference on Web Information Systems and Technologies (WEBIST 2016), vol. 2, 2016, pp. 243-250.

[9] Kairos, "Emotions Analysis API, Detection \& Analyze Emotions," 2017, online available at https://www.kairos.com/emotion-analysis-api.

[10] R. Plutchik, "The nature of emotions human emotions have deep evolutionary roots, a fact that may explain their complexity and provide tools for clinical practice," American scientist, vol. 89, no. 4, pp. 344350, 2001.

[11] J. Posner, J. A. Russell, and B. S. Peterson, "The circumplex model of affect: An integrative approach to affective neuroscience, cognitive development, and psychopathology," Development and psychopathology, vol. 17, no. 03, pp. 715-734, 2005.

[12] Microsoft, "Kinect for Windows," 2017, online available at https:// developer.microsoft.com/en-us/windows/kinect.

[13] _ _ "Speech Basics-WPF C\# Sample," 2017, online available at https: //msdn.microsoft.com/en-us/library/hh855387.aspx. 
[14] — , "SpeechSynthesizer," 2016, online available https://msdn.microsoft.com/en-us/library/system.speech.synthesis. speechsynthesizer(v=vs.110).aspx.

[15] T. De Pessemier, S. Dooms, and L. Martens, "Comparison of group recommendation algorithms," Multimedia tools and applications, vol. 72, no. 3, pp. 2497-2541, 2014.

[16] Microsoft, "Slideshow Gestures-WPF C\# Sample," 2017, online available at https://msdn.microsoft.com/en-us/library/jj131042.aspx.

[17] M. Rosack, "A facial recognition implementation for the Kinect for Windows API," 2015, online available at https://github.com/mrosack/ Sacknet.KinectFacialRecognition.

[18] P. Ekman, W. V. Friesen, M. O'Sullivan, A. Chan, I. DiacoyanniTarlatzis, K. Heider, R. Krause, W. A. LeCompte, T. Pitcairn, P. E. RicciBitti et al., "Universals and cultural differences in the judgments of facial expressions of emotion." Journal of personality and social psychology, vol. 53, no. 4, p. 712, 1987.

[19] H. A. Alabbasi, P. Moldoveanu, and A. Moldoveanu, "Real time facial emotion recognition using kinect v2 sensor," IOSR J. Comput. Eng. Ver. II, vol. 17, no. 3, pp. 2278-2661, 2015.

[20] Pandas, "Python Data Analysis Library," 2016, online available at http: //pandas.pydata.org/.

[21] F. Pedregosa, G. Varoquaux, A. Gramfort, V. Michel, B. Thirion, O. Grisel, M. Blondel, P. Prettenhofer, R. Weiss, V. Dubourg, J. Vanderplas, A. Passos, D. Cournapeau, M. Brucher, M. Perrot, and E. Duchesnay, "Scikit-learn: Machine learning in Python," Journal of Machine Learning Research, vol. 12, pp. 2825-2830, 2011.

[22] Scikit-learn developers, "RBF SVM parameters," 2016, online available at http://scikit-learn.org/stable/auto_examples/svm/plot_rbf_parameters. html.

[23] V. Pterneas, "Finger Tracking using Kinect v2," 2016, online available at https://www.codeproject.com/Articles/1074037/ Finger-Tracking-using-Kinect-v. 